\title{
Combination of HSV1-TK/shTERT by retrovirus vector inhibits hepatocellular carcinoma cell growth in vitro and in vivo
}

\author{
HONGSHENG LI ${ }^{1}$, QIAOYU WANG ${ }^{1}$, DONGLI LI ${ }^{2}$, QUANSHI WANG ${ }^{1}$ and HUBING WU ${ }^{1}$ \\ ${ }^{1}$ NanFang PET Center and ${ }^{2}$ Department of Medical Imaging Center, Nanfang Hospital, \\ Southern Medical University, Guangzhou, Guangdong 510515, P.R. China
}

Received October 8, 2014; Accepted November 20, 2014

DOI: $10.3892 /$ or.2014.3697

\begin{abstract}
Hepatocellular carcinoma (HCC) is a common malignant carcinoma worldwide and the third leading cause of cancer mortality. However, current treatment strategies are not potent enough to combat this disease. Therefore, identification of novel and more effective treatments is crucial. Of the current methods, gene therapy, which targets cancer-specific expression and limits toxicity, is a new strategy for treating cancers. In this study, we developed a retroviral vector containing herpes simplex virus type-1-thymidine kinase (HSV1-TK) and a short hairpin RNA for the human telomerase reverse transcriptase (hTERT) gene and investigated the antitumor effects in an in vitro and in vivo mouse model of liver cancer, monitored by PET image. In vitro experiments on HCC cells in the TK-shTERT treatment group showed significant accumulation of ${ }^{18} \mathrm{~F}-\mathrm{FHBG}$, which preferentially inhibits HCC cell growth with extremely limited toxicity in normal cells. In vivo studies showed a significant reduction of growth in the TK-shTERT treatment group. In conclusion, these findings showed that combination HSV1-TK/hTERT gene therapy effectively and safely inhibits HCC cell growth in vitro and in vivo and is worthy of development in clinical trials for the treatment of HCC.
\end{abstract}

\section{Introduction}

Hepatocellular carcinoma (HCC) is the fifth most common malignant carcinoma worldwide and the third leading cause of cancer mortality $(1,2)$. Although its mortality decreased along with great advances in surgical resection and chemotherapy, the long-term prognosis remains unsatisfactory $(3,4)$. Thus, effective and innovative therapeutic procedures are required to combat this disease. Evidence has shown that in addition

Correspondence to: Dr Quanshi Wang, NanFang PET Center, Nanfang Hospital, Southern Medical University, 1838 Guangzhou Road, Guangzhou, Guangdong 510515, P.R. China

E-mail: quanshiwang56@sogou.com

Key words: HSV1-thymidine kinase, hTERT, cell growth, hepatocellular carcinoma to current chemotherapy, gene therapy using small-interfering RNA (RNAi) is a potent therapeutic approach in HCC therapy (5).

Telomerase is a ribonucleoprotein complex that is involved in tumor growth and progression in part through maintaining the ends of chromosomes (6). Human telomerase reverse transcriptase (hTERT), an essential subunit of the telomerase complex, maintains the length of telomeres by reverse transcription and the addition of TTAGGG repeats onto the telomeric ends of the chromosomes (7). Moreover, in various human cancer cells, such as liver and ovarian cancer cells, a close correlation between hTERT expression and telomerase activity has been identified (8-10). Previous findings showed that the hTERT appeared to be the major determinant of telomerase activity (11). These observations indicated that hTERT expression and telomerase activity serve as useful diagnostic and/or prognostic markers in many types of human malignancies. Accordingly, the potential of telomerase inhibition by targeting hTERT as an effective therapy for cancer treatment has been demonstrated $(12,13)$. hTERT was previously found to be expressed in most cancer cells but not in normal cells (14). However, previous results showed that hTERT can be detected in both malignant and normal tissues (15-17). Thus, to use this target by silencing hTERT expression in the treatment of cancer requires identification of a method with tumor cell-specificity and low toxicity, which can effectively inhibit hTERT gene expression in tumor cells but cannot affect hTERT activity in normal immortal cells.

Different approaches are currently under investigation to develop gene therapy of cancer. However, experience from clinical trials of cancer gene therapy indicates that no single therapeutic strategy can effectively eradicate cancer, whereas combined poly-gene therapy is a more reliable approach to combating cancer. A well-established radionuclide-based reporter gene system is the herpes simplex virus type-1-thymidine kinase (HSV1-TK) enzyme, which can specifically infect a variety of cancer cells but not normal mammalian cells $(18,19)$. This reporter gene can itself be the therapeutic gene, and anticancer gene therapy using HSV1-TK can be coupled with imaging of the accumulation of radio-labeled probes such as 9-[4-fluoro-3-(hydroxymethyl)butyl]guanine (FHBG) or 5-iodo-20-fluoro-20deoxy-1-b-D-arabinofuranosyluracil (FIAU) $(20,21)$, which can be imaged using PET. Thus, co-expression of a reporter gene and a therapeutic gene 
may allow for delivery of therapeutic gene to cancer cells efficiently and safely and treat cancer effectively. Consequently, we constructed triple-expressing vector in which the HSV1-TK gene and shTERT were driven by the pLXSN and the U6 promoters and detected the antitumor effects on cells in vitro as well as tumor inhibition in vivo. In this study, the combination of hTERT shRNA/HSV1-TK preferably targeted HCC cells and significantly suppressed the tumor growth in liver tumor xenograft. Moreover, hTERT shRNA/HSV1-TK showed virtually no toxicity in normal cells, suggesting that the hTERT shRNA/HSV1-TK vector may be exploited as a potential new therapeutic strategy for HCC.

\section{Materials and methods}

Cell lines and culture. Human HCC cell lines (BEL-7402, HCC36, HepG2, HA22T and Hep3B), normal mammary epithelial (L-02, QSG-7701), and normal lung fibroblasts (WI-38) were purchased from the American Type Culture Collection (Manassas, VA, USA) and maintained in Dulbecco's modified Eagle's medium (DMEM) supplemented with $10 \%$ fetal bovine serum, $100 \mathrm{U} / \mathrm{ml}$ penicillin, $100 \mu \mathrm{g} / \mathrm{ml}$ streptomycin. The cells were maintained at $37^{\circ} \mathrm{C}$ in a humidified atmosphere containing $5 \% \mathrm{CO}_{2} / 95 \%$ air.

Construction of recombinant retroviral vectors and vector titration. The siRNA sequence targeting hTERT corresponded to the coding region from 385 to 393 : 5'-AAGCACTTCCTCT ACTCCTCA-3'. The oligonucleotides with a sequence predicted to induce efficient small-interfering RNA (RNAi) of hTERT (containing sense and antisense sequences linked by the hairpin loop: CGAA) were synthesized as follows: Forward: 5'-CGTCGACGCACTTCCTCTACTCCTCATT CAAGAGATGAGGA-3' and reverse: 5'-CAAGCTTCTCGA GTCTAGAAAAAGCACTTCCTCTACTCCTCATCTC-3'. These oligonucleotides were annealed in STE buffer $(10 \mathrm{mM}$ Tris pH 8.0, $50 \mathrm{mM} \mathrm{NaCl}, 1 \mathrm{mM}$ EDTA) at $94^{\circ} \mathrm{C}$ for $5 \mathrm{~min}$ and cooled gradually. The double-stranded product was cloned downstream to the human U6 promoter of the pGEM/U6 vector (Genscripts). The recombinant retroviral vector $\mathrm{pLXSN}$ (Clontech) was employed to develop the pLXSN-TK-U6shTERT vector. Full-length HSV1-TK cDNA (kindly provided by Dr Qiu Xinfang, FuDan University, Shanghai, China) sequence coupled to U6 promoter was removed from pRNATshTERT vector, and a NheI-SalI restriction site was introduced into the pRNAT-shTERT vector by PCR. HSV1-TK cDNA was amplified by PCR using the sense primer, which contains a NheI restriction site, and the anti-sense primer, which contains a SalI restriction site. Following restriction digestion with NheI and SalI of pRNAT-hTERT shRNA, the HSV1-TK sequence was ligated into the pRNAT-shTERT vector. The resulting vector containing shTERT sequence and HSV1-TK cDNA coupled to the U6, was designated as pLXSN-TK-U6shTERT vector, and confirmed by DNA sequencing. A retroviral vector containing the shTERT sequence ( $\mathrm{pLXSN}$ U6-hTERT shRNA) was also developed. The U6 promoter was inserted into the vector as described above.

Plasmid vectors were transfected into the amphotropic packaging cell line 293 by using the calcium phosphate transfection system (Invitrogen Life Technologies, Carlsbad,
CA, USA) reagents, as previously described (22). Transfected cells were selected in a medium containing G418 (Invitrogen) and single cell-derived clones were isolated and expanded to cell lines. Viral titer, determined by infection of NIH3T3 with virus-containing supernatants from single cell-derived clones of 293 producer cells as described previously (22), ranged from $10^{4}$ to $10^{6} \mathrm{cfu} / \mathrm{ml}$. The supernatant from the producer cell clones with higher viral titer was used to transduce target cells.

MicroPET imaging of ${ }^{18} F-F H B G$ in vivo. When flank tumors reached $200 \mathrm{~mm}^{3}$ in size, $0.2 \mathrm{ml}$ pLXSN-U6-shTERT $\left(5 \times 10^{5} \mathrm{pfu} / \mathrm{ml}\right)$ or $0.2 \mathrm{ml} \mathrm{pLXSN}-\mathrm{TK}-\mathrm{U} 6$-shTERT $\left(5 \times 10^{5} \mathrm{pfu} / \mathrm{ml}\right)$ were injected directly into the center of the tumor. Mice were anesthetized via inhalation of isoflurane (1-1.5\%) with an oxygen flow rate of $21 / \mathrm{min}$. Depth of anesthesia was monitored by respiratory rate and eye and footpad reflex. A total of $150 \mathrm{MBq}(0.2 \mathrm{ml}){ }^{18} \mathrm{~F}$-FHBG was injected into the tail vein to acquire dynamic volumetric data for $210 \mathrm{~min}$. Volumetric images were reconstructed with filtered back projection after the data were corrected for uniformity, scatter, attenuation, decay, and injected activity using the software AsiPro 4.1 provided by the manufacturer. Time-activity curves were generated from the selected regions of interest including pLXSN-U6-shTERT (on the left flank), pLXSN-TK-U6shTERT tumor (on the right flank).

Western blot analysis. The cells were lysed in mammalian protein extraction reagent (Pierce Biotechnology, Rockford, IL, USA). Cell lysates were collected and protein concentration of the cell lysates was measured. Proteins (10-20 $\mu \mathrm{g})$ were resolved by SDS-polyacrylamide gel electrophoresis and transferred to PVDF membranes (Bio-Rad, Hercules, CA, USA). The membranes were then incubated with primary antibodies in $3 \%$ bovine serum albumin/Tris-buffered saline/Tween-20 at $4^{\circ} \mathrm{C}$ overnight, followed by incubation with secondary antibodies at room temperature for $1 \mathrm{~h}$. The protein signals were detected by ECL method. Western blotting reagents were obtained from Pierce Biotechnology. The antibodies to hTERT and $\beta$-actin were purchased from Cell Signaling Technology (Danvers, MA, USA).

Cell viability assay. Cell viability was measured by 3-(4,5-dimethylthiazol-2-yl)-2,5-diphenyltetrazolium bromide (MTT) assay. Briefly, the cells were plated at a density of $5 \times 10^{3}$ cells/well in 96-well tissue culture plates and subjected to different treatments. Following a $72-\mathrm{h}$ incubation at $37^{\circ} \mathrm{C}$ in a humidified atmosphere containing $5 \% \mathrm{CO}_{2} / 95 \%$ air, the cells were incubated for another $4 \mathrm{~h}$ with MTT reagent. The formazan product was dissolved in dimethyl sulfoxide and read at $570 \mathrm{~nm}$ on a Victor 3 Multi Label plate reader (PerkinElmer, Boston, MA, USA).

In vivo combination therapy. When tumor masses reached average volume of $\sim 200 \mathrm{~mm}^{3}$, pLXSN-U6-shTERTand pLXSN-TK-U6-shTERT were initiated. Tumor xenograft mice were randomly divided into the control, pLXSN-U6-shTERT and pLXSN-TK-U6-shTERT groups. The mice in each group $(n=5)$ received intratumor injections of either $0.2 \mathrm{ml} \mathrm{pLXSN}$ U6-shTERT $\left(5 \times 10^{5} \mathrm{pfu} / \mathrm{ml}\right)$ or $0.2 \mathrm{ml}$ pLXSN-TK-U6-shTERT $\left(5 \times 10^{5} \mathrm{pfu} / \mathrm{ml}\right)$ on continuous injection for five days. The mice 
A
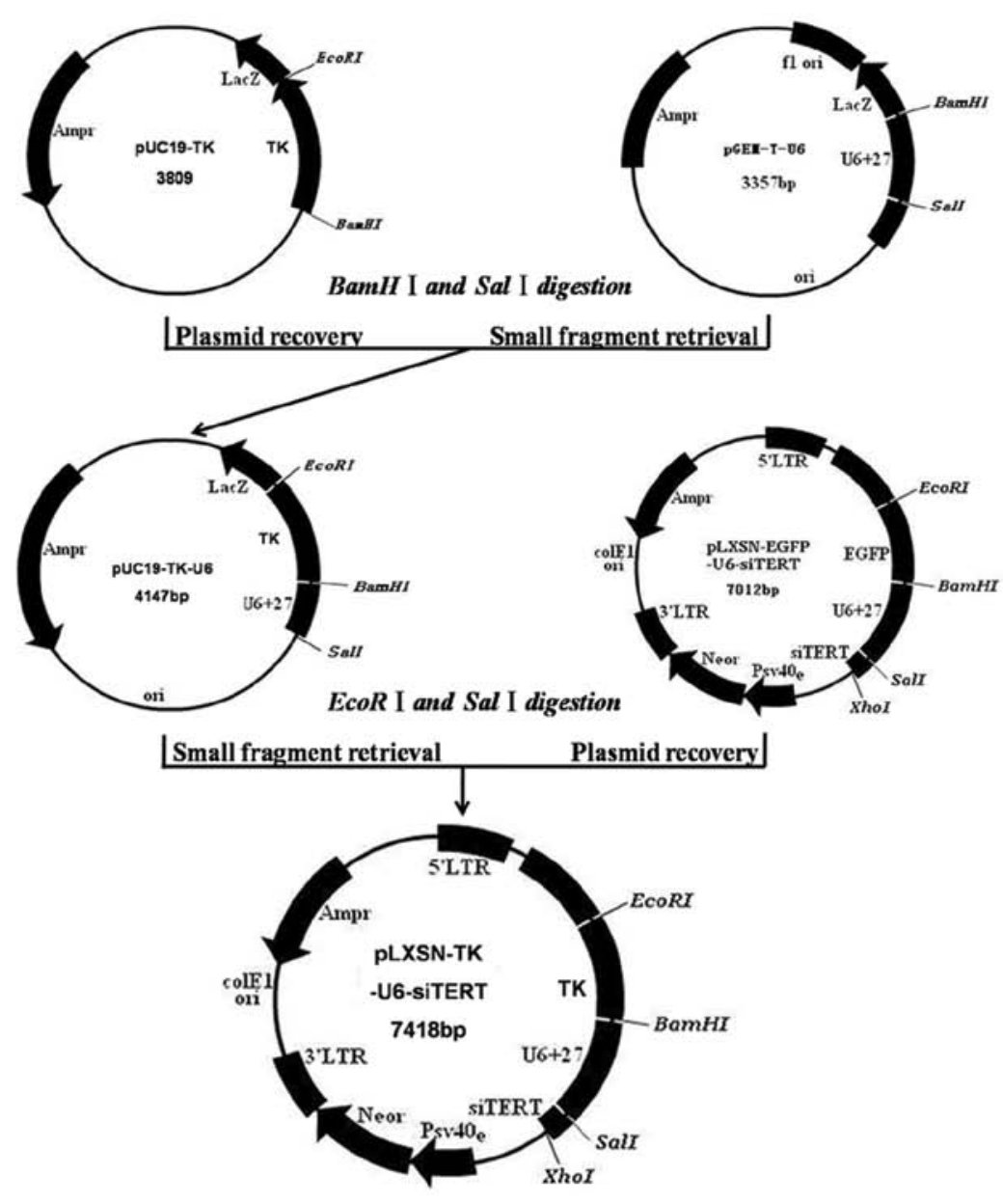

B

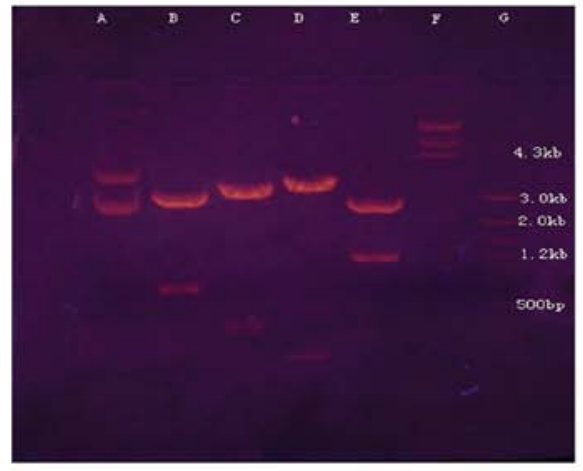

C

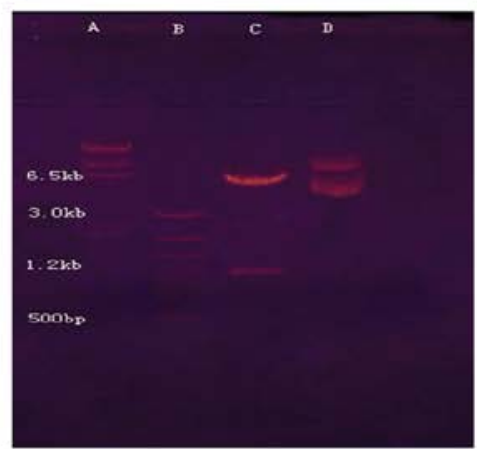

D

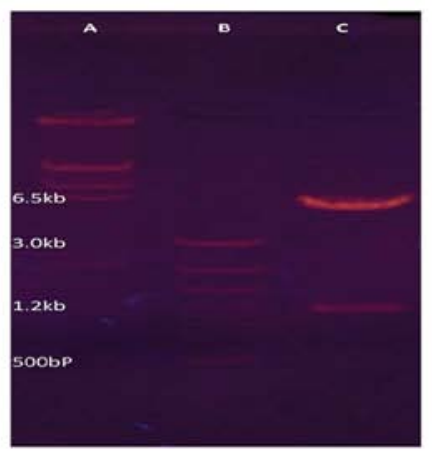

Figure 1. Successful construction and identification of recombinant plasmids. (A) The schema of the pLXSN-TK-U6-shTERT recombinant plasmid construction. (B) Identification of pUC19-EGFP-U6-shTERT vector. Lane A, control pUC19/EGFP-U6-shTERT; lane B, pUC19/EGFP-U6-shTERT/EcoRI, BamHI; lane C, pUC19/EGFP-U6-shTERT/BamHI, SalI; lane D, pUC19/EGFP-U6-shTERT/SalI, XhoI; lane E, pUC19/EGFP-U6-shTERT/EcoRI, XhoI; lane F, $\lambda$ DNA/ HindIII markers; lane G, 100 bp DNA Ladder. (C) Identification of pLXSN-EGFP-U6-shTERT vector. Lane A, $\lambda$ DNA/HindIII markers; lane B, 100 bp DNA Ladder; lane C, pLXSN/EGFP-U6-shTERT/EcoRI, XhoI; lane D, control pLXSN/EGFP-U6-shTERT. (D) Identification of pLXSN/TK-U6-shTERT. Lane A, $\lambda$ DNA/HindIII markers; lane B, pLXSN/TK-U6-shTERT/EcoRI, BamHI.

in the control group received saline injections at the same time. Individual tumor size was measured every 2 days by use of a caliper and tumor volumes were determined by measuring the length (L) and width (W) of the tumors and calculating using the following formula: $\mathrm{V}=\mathrm{LW}^{2} / 2$. Animal maintenance and experimental procedures were approved by the Nanfang Hospital Animal Ethics Committee.

In vivo apoptosis assays. At the end of the experiment (5 weeks after intratumor injection), the mice were humanely euthanized and tumors were surgically dissected. The tumor specimens were fixed in $4 \%$ paraformaldehyde for TUNEL staining (Roche Applied Sciences, Indianapolis, IN, USA) according to the manufacturer's instructions. Briefly, the slides were incubated with $50 \mathrm{ml}$ of TUNEL reaction mixture in a humidified atmosphere for $1 \mathrm{~h}$ at $37^{\circ} \mathrm{C}$ in the dark. The percentage of TUNEL apoptotic cells was analyzed by randomly selecting five independent fields for each sample.
Statistical analysis. The results are given as mean \pm SD. Student's t-test was used to analyze the significance of differences. The significance level was set at $\mathrm{P}<0.05$.

\section{Results}

Construction and identification of pLXSN-TK-U6-shTERT recombinant plasmid. Recombinant plasmids pGEM-TshTERT, pGEM-T-U6, pUC19-EGFP and pUC19-TK were constructed. BamHI/SalI and SalI/HindIII double enzyme digestion methods were selected to obtain pUC19-EGFP-U6 and pUC19-EGFP-U6-shTERT respectively, EcoRI/XhoI double enzyme digested pUC19-EGFP-U6-shTERT and pLXSN plasmids to obtain pLXSN-EGFP-U6-shTERT, BamHI/ SalI double enzyme digested pGEM-T-U6 and pUC19-TK plasmids to obtain pUC19-TK-U6, and SalI/EcoRI double enzyme-digested recombinant plasmids pLXSN-EGFP-U6shTERT and pUC19-TK-U6 to obtain pLXSN-TK-U6-shTERT 


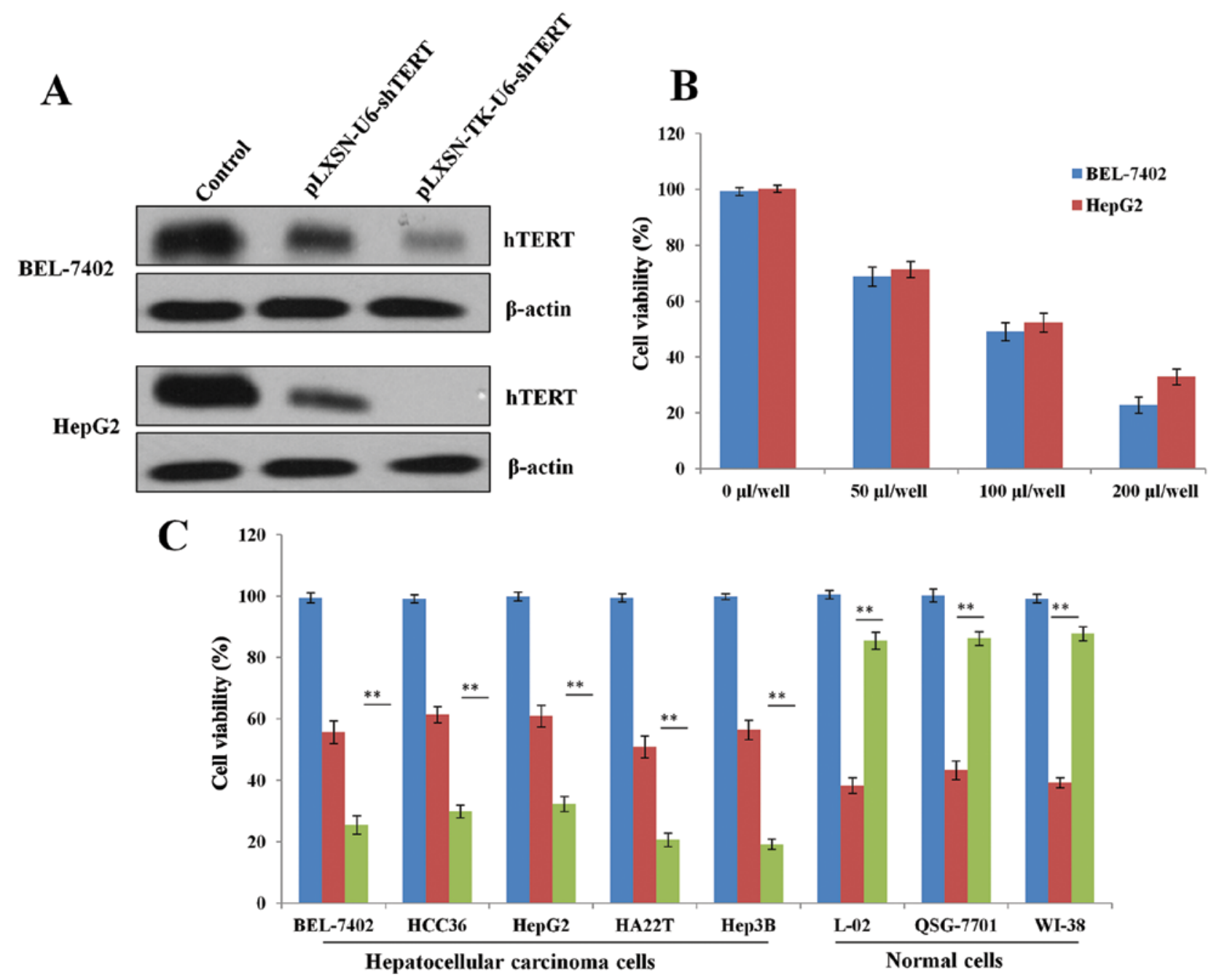

Figure 2. pLXSN-TK-U6-shTERT recombinant plasmid effectively and preferentially kills liver cancer cells in vitro. (A) hTERT expression was detected in BEL-7402 cell lysate after $48 \mathrm{~h}$ of transient transfection by western blot analysis. (B) BEL-7402 and HepG2 cells were cotransfected with increasing amount $(0,50,100$ or $200 \mu 1 /$ well) of pLXSN-TK-U6-shTERT. (C) In vitro cell killing activities of pLXSN-TK-U6-shTERT (200 $\mu 1 /$ well) or pLXSN-U6-shTERT $\left(200 \mu 1 /\right.$ well) in liver cancer cells and normal cells were measured by MTT assay. Data shown are representative of three identical experiments. ${ }^{* *} \mathrm{P}<0.01, \mathrm{t}$-test.

(Fig. 1A). Restriction enzyme identification of recombinant plasmids are shown in Fig. 1B.

pLXSN-TK-U6-shTERT recombinant plasmid preferentially inhibits HCC cell growth in vitro with extremely limited toxicity in normal cells. To determine whether the pLXSNTK-U6-shTERT plasmid can specifically target HCC cells, the expression of hTERT was detected by western blotting $48 \mathrm{~h}$ after transient transfection in BEL-7402 and HepG2 HCC cells (Fig. 2A). The results showed that pLXSN-TK-U6shTERT inhibited cell growth in vitro in a dose-dependent manner (Fig. 2B). Moreover, we examined the killing effects of pLXSN-TK-U6-shTERT in a panel of HCC and normal cell lines. The results showed that the pLXSN-TK-U6-shTERT inhibited cell growth more effectively than pLXSN-U6shTERT in vitro (Fig. 2B). However, in normal cells, the cell killing activity of pLXSN-U6-shTERT was more potent than that of pLXSN-TK-U6-shTERT (Fig. 2C). Thus, the cytotoxic effect of pLXSN-TK-U6-shTERT is potent in cancer cells but limited in normal cells, indicating that pLXSN-TK-U6shTERT is tumor-specific in vitro and is a potential therapeutic agent that can be used for the treatment of HCC.
Detection of anatomic sites of ${ }^{18} \mathrm{~F}-\mathrm{FHBG}$ sequestration with microPET. When tumor masses reached an average volume of $\sim 200 \mathrm{~mm}^{3}$, the mice received intratumoral injections of $0.2 \mathrm{ml}$ pLXSN-U6-shTERT ( $5 \times 10^{5} \mathrm{pfu} / \mathrm{ml}$, left flank) or $0.2 \mathrm{ml}$ pLXSN-TK-U6-shTERT ( $5 \times 10^{5} \mathrm{pfu} / \mathrm{ml}$, right flank). Compared with shTERT tumors (left flank), TK-shTERT tumors of mice showed significant accumulation (right flank) over time as a result of intracellular entrapment of HSV-TK-phosphorylated ${ }^{18} \mathrm{~F}-\mathrm{FHBG}, 150 \mathrm{~min}$ after ${ }^{18} \mathrm{~F}-\mathrm{FHBG}$ injection, when the uptake value reached its greatest level (Fig. 3A and B). The liver and kidney concentration of ${ }^{18} \mathrm{~F}-\mathrm{FHBG}$ was significantly greater, and no radioactive distribution was observed in the brain (Fig. 3B).

pLXSN-TK-U6-shTERT recombinant plasmid exerts a significant antitumor effect in mouse xenograft model of HCC. To investigate the therapeutic effects of pLXSN-TK-U6-shTERT in vivo, we established a mouse xenograft model with the BEL-7402 HCC cell line. When tumor masses reached an average volume of $\sim 400 \mathrm{~mm}^{3}$, the mice in each group received intratumoral injections of pLXSN-U6-shTERT plasmid or pLXSN-TK-U6-shTERT plasmid of continuous injection for 

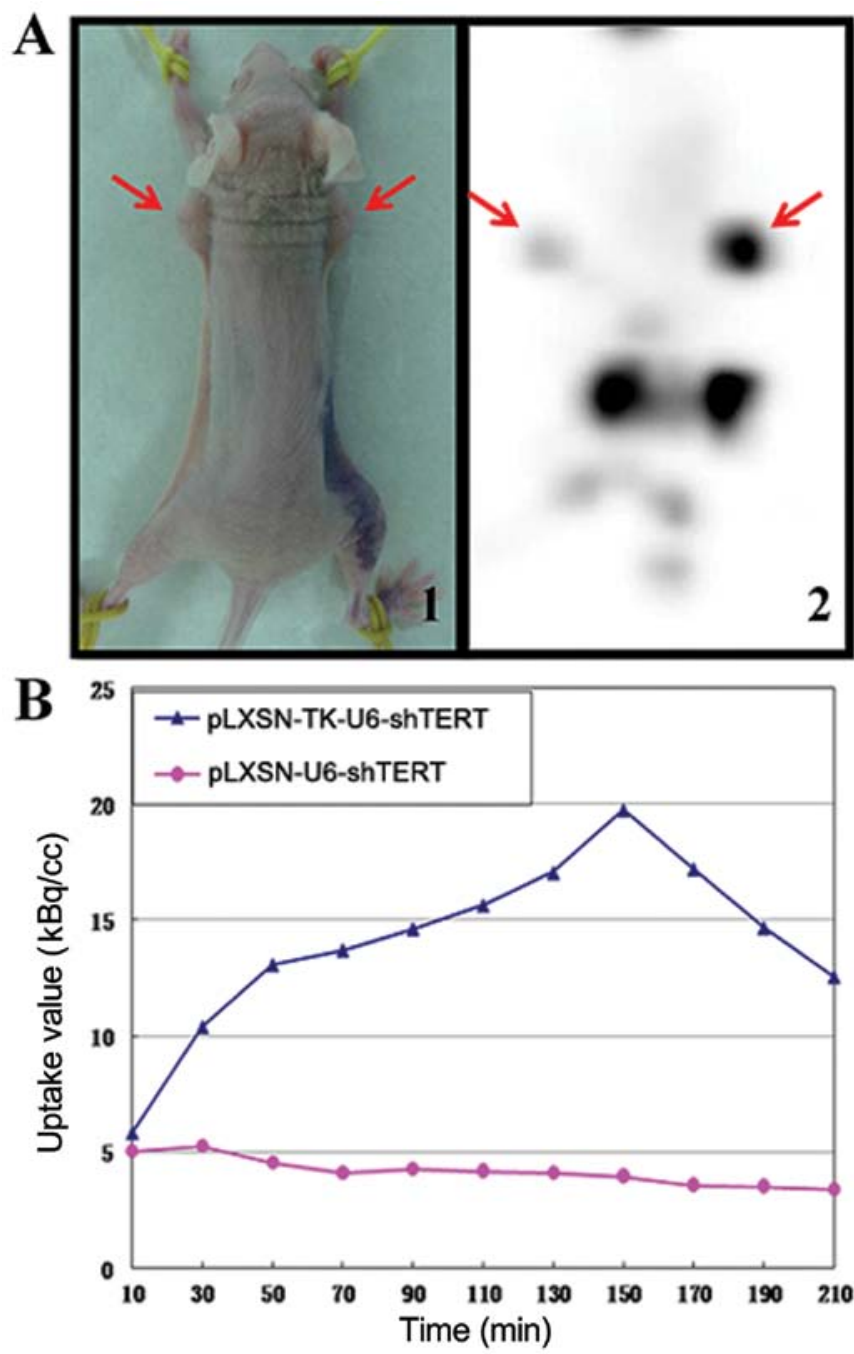

Figure 3. In vivo animal imaging with microPET using ${ }^{18} \mathrm{~F}-\mathrm{FHBG}$ during in vivo therapy. (A) MicroPET images with simultaneously captured images reveal significant tracer accumulation in pLXSN-TK-U6-shTERT tumors, as compared with pLXSN-U6-shTERT tumors. (B) Uptake value of pLXSN-TK-U6-shTERT tumors increased and reached the greatest level at $150 \mathrm{~min}$, while the uptake value of pLXSN-U6-shTERT group mice slightly decreased.

5 days. Five weeks after the first treatment, the pLXSN-TK-U6shTERT group significantly inhibited tumor growth compared with the pLXSN-U6-shTERT group (Fig. 4A and B). These findings were further supported by the increase in apoptosis and decrease in proliferation in the tumors of the pLXSN-TK-U6shTERT-treated groups. Notably, although the two treatment groups showed a statistically significant trend of increase in TUNEL-positive cells and decrease in Ki67-positive cells compared with the control, pLXSN-TK-U6-shTERT showed significantly higher activities than pLXSN-U6-hTERT shRNA, suggesting that pLXSN-TK-U6-shTERT has higher targeting power over pLXSN-U6-shTERT (Fig. 4C and D). Collectively, these results showed that pLXSN-TK-U6-shTERT consistently exerts strong antitumor effects on liver tumor in vivo and induces apoptosis with high-tumor specificity.

\section{Discussion}

Cancer cells frequently overexpress hTERT, a determinant of telomerase activity, resulting in enhanced proliferation and tumor progression $(23,24)$. hTERT associates with human telomeres and may enhance genomic stability and DNA repair in human cancer cells (25). Thus, hTERT is an attractive therapeutic target in malignant tumor treatment. A variety of gene-targeting approaches were found to interfere with hTERT function (26-28). Among them, RNAi-mediated hTERT gene silencing provides an efficient method to inhibit telomerase activity for human cancer therapy (29-31). RNAi is known to be very effective and selective in vitro, however, issues, including incomplete suppression of target genes, requirement of cytotoxic transfection reagents and/or enhancers, positively impairing normal cellular functions and lack of effective delivery system, hamper the development of this novel therapy for cancer treatment. Thus, the development of low-toxic and potent shRNA delivery systems is a crucial step for the success of RNAi-based cancer therapy.

The most studied suicide gene is HSV1-TK. HSV-TKbased suicide gene therapy has been used to target cancers and its role has been assessed in several clinical trials. The reporter gene can itself be the therapeutic gene or can be coupled with the therapeutic gene (32). Clinical trials using this approach have been conducted in patients with gliomas and no serious adverse events were reported (33). The successful employment of several candidate gene 

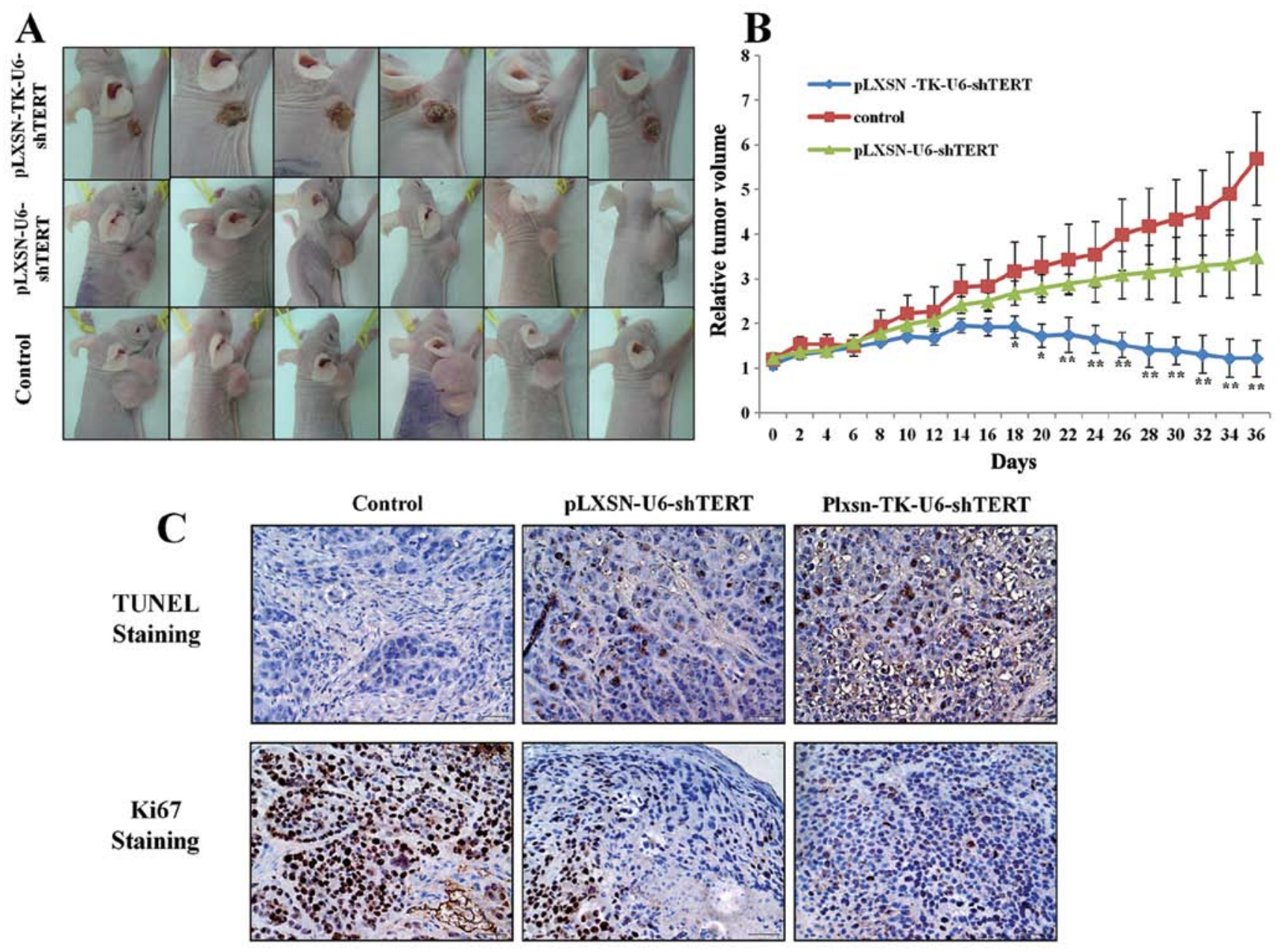

Figure 4. pLXSN-TK-U6-shTERT recombinant plasmid inhibits tumor growth more effectively than pLXSN-U6-shTERT in a mouse xenograft model of liver cancer. (A) Tumor size in mice treated with pLXSN-TK-U6-shhTERT or pLXSN-U6-shhTERT. Tumor size was measured every 2 days after treatment. Each point is the mean $\pm \mathrm{SD}$. Results shown are representative of three identical experiments ${ }^{*} \mathrm{P}<0.05$ and ${ }^{* *} \mathrm{P}<0.01$, t-test. (B) TUNEL staining in paraffin sections of the tumors. (C) Expression of the cell proliferation marker Ki67 in paraffin sections of the tumors. Scale bar, $50 \mu \mathrm{m}$.

combinations for HSV1-TK suicide gene therapy have been reported including IL-2 (34), STAT3 (35), cytosine deaminase (36), nitroreductase (37), and carboxylesterase (38). Non-invasive imaging should be accompanied with gene therapy approaches for treatment response monitoring as well as for assessment of distribution, extent and duration of transgene expression. Rapid washout of activity from the blood significantly decreased sensitivity and specificity of tracer accumulation in HSV1-TK-expressing tumors. ${ }^{18} \mathrm{~F}$-FHBG has emerged as the most reliable agent for treatment response monitoring. In this study, we demonstrated the applicability of non-invasive imaging using ${ }^{18} \mathrm{~F}$-FHBG for monitoring cancer gene therapy in an experimental animal model of HSV1-TK-expressing tumor xenografts, which is also in concordance with a previous report (39). In the present study, we demonstrated the in vitro and in vivo therapeutic efficacy of retroviral vector-mediated combined HSV1-TK suicide and hTERT gene therapy for HCC. The use of an LXSN-TK-based retroviral vector allows high transfection efficiency of hTERT expression and selective targeting of cancer cells, while sparing normal cells in vitro.

Although retrovirus vectors increasingly receiving attention in the field of liver cancer gene therapy due to their hepatic tropism and high titers, they have one major drawback of in vivo delivery and transduction $(40,41)$, which may be relatively to obtain in liver cancer in which percutaneous locoregional treatment is easy to perform. In the current study, we also carried out in vivo transduction by repeated intratumoral injection of the vector. When tumors were transduced in vivo with TK-shTERT retroviral vector, we observed a strong antitumor effect, with complete regression of tumors composed of transduced cells compared with the shTERT group, showing wide apoptotic areas and markedly decreased Ki67-positive cells in the residual tumor (Fig. 4), thus confirming the efficacy of the vector and the possibility to transduce a sufficient amount of tumor cells by locoregional treatment, as also confirmed by the in vivo experiment.

Taken together, our study has demonstrated enhanced antitumor effects by the use of combination gene therapy using TK-shTERT for tumor xenografts in mice as compared with shTERT single therapeutic approach. Furthermore, therapeutic response monitoring was possible by serial non-invasive in vivo imaging using a reporter gene system. These results suggest that shRNA targeting hTERT, coupled with the HSV-TK suicide gene, may be therapeutically useful for HCC and potentially for other malignancies. 


\section{Acknowledgements}

This study was funded by the National Natural Science Foundation of China and the grant numbers are 30370426 and 81071174.

\section{References}

1. Roberts LR: Sorafenib in liver cancer - just the beginning. N Engl J Med 359: 420-422, 2008.

2. Llovet JM: Updated treatment approach to hepatocellular carcinoma. J Gastroenterol 40: 225-235, 2005.

3. Pang RW, Joh JW, Johnson PJ, Monden M, Pawlik TM and Poon RT: Biology of hepatocellular carcinoma. Ann Surg Oncol 15: 962-971, 2008.

4. Yang LY, Fang F, Ou DP, Wu W, Zeng ZJ and Wu F: Solitary large hepatocellular carcinoma: a specific subtype of hepatocellular carcinoma with good outcome after hepatic resection. Ann Surg 249: 118-123, 2009.

5. Gao J, Chen H, Yu Y, et al: Inhibition of hepatocellular carcinoma growth using immunoliposomes for co-delivery of adriamycin and ribonucleotide reductase M2 siRNA. Biomaterials 34 10084-10098, 2013.

6. Kim NW, Piatyszek MA, Prowse KR, et al: Specific association of human telomerase activity with immortal cells and cancer. Science 266: 2011-2015, 1994.

7. Feng WD J, Funk SS, Wang SL, et al: The RNA component of human telomerase. Science 269: 1236-1241, 1995.

8. Zhang PH, Zou L and Tu ZG: RNAi-hTERT inhibition hepatocellular carcinoma cell proliferation via decreasing telomerase activity. J Surg Res 131: 143-149, 2006.

9. Sun PM, Wei LH, Luo MY, et al: The telomerase activity and expression of hTERT gene can serve as indicators in the anticancer treatment of human ovarian cancer. Eur J Obstet Gynecol Reprod Biol 130: 249-257, 2007.

10. Toshikuni N, Nouso K, Higashi T, et al: Expression of telomerase-associated protein 1 and telomerase reverse transcriptase in hepatocellular carcinoma. Br J Cancer 82: 833-837, 2000.

11. Beattie TL, Zhou W, Robinson MO and Harrington L: Reconstitution of human telomerase activity in vitro. Curr Biol 8 : 177-180, 1998.

12. Herbert B, Pitts AE, Baker SI, Hamilton SE, Wright WE, Shay JW and Corey DR: Inhibition of human telomerase in immortal human cells leads to progressive telomere shortening and cell death. Proc Natl Acad Sci USA 96: 14276-14281, 1999.

13. Folini $\mathrm{M}$ and Zaffaroni N: Targeting telomerase by antisensebased approaches: perspectives for new anti-cancer therapies. Curr Pharm Des 11: 1105-1117, 2005.

14. Meyerson M, Counter CM, Eaton EN, et al: hEST2, the putative human telomerase catalytic subunit gene, is up-regulated in tumor cells and during immortalization. Cell 90: 785-795, 1997.

15. Pannone G, De Maria S, Zamparese R, et al: Prognostic value of human telomerase reverse transcriptase gene expression in oral carcinogenesis. Int J Oncol 30: 1349-1357, 2007.

16. Li W, Li L, Liu Z, et al: Expression of the full-length telomerase reverse transcriptase (hTERT) transcript in both malignant and normal gastric tissues. Cancer Lett 260: 28-36, 2008.

17. Nagao K, Tomimatsu M, Endo H, Hisatomi $\mathrm{H}$ and Hikiji $\mathrm{K}$ : Telomerase reverse transcriptase $m R N A$ expression and telomerase activity in hepatocellular carcinoma. J Gastroenterol 34: 83-87, 1999.

18. Gil Z, Rein A, Brader P, Li S, Shah JP, Fong Y and Wong RJ: Nerve-sparing therapy with oncolytic herpes virus for cancers with neural invasion. Clin Cancer Res 13: 6479-6485, 2007.

19. Kelly K, Brader P, Rein A, Shah JP, Wong RJ, Fong Y and Gil Z: Attenuated multimutated herpes simplex virus-1 effectively treats prostate carcinomas with neural invasion while preserving nerve function. FASEB J 22: 1839-1848, 2008.

20. Tjuvajev JG, Joshi A, Callegari J, et al: A general approach to the non-invasive imaging of transgenes using cis-linked herpes simplex virus thymidine kinase. Neoplasia 1: 315-320, 1999.
21. Gambhir SS, Barrio JR, Phelps ME, et al: Imaging adenoviraldirected reporter gene expression in living animals with positron emission tomography. Proc Natl Acad Sci USA 96: 2333-2338, 1999.

22. Barzon L, Bonaguro R, Castagliuolo I, et al: Gene therapy of thyroid cancer via retrovirally-driven combined expression of human interleukin-2 and herpes simplex virus thymidine kinase. Eur J Endocrinol 148: 73-80, 2003.

23. Bertorelle R, Briarava M, Rampazzo E, et al: Telomerase is an independent prognostic marker of overall survival in patients with colorectal cancer. Br J Cancer 108: 278-284, 2013.

24. Liu H, Liu S, Wang H, et al: Genomic amplification of the human telomerase gene (hTERC) associated with human papillomavirus is related to the progression of uterine cervical dysplasia to invasive cancer. Diagn Pathol 7: 147, 2012.

25. Sharma GG, Gupta A, Wang H, et al: hTERT associates with human telomeres and enhances genomic stability and DNA repair. Oncogene 22: 131-146, 2003.

26. Kraemer K, Fuessel S, Kotzsch M, et al: Chemosensitization of bladder cancer cell lines by human telomerase reverse transcriptase antisense treatment. J Urol 172: 2023-2028, 2004.

27. Hao ZM, Luo JY, Cheng J, et al: Intensive inhibition of hTERT expression by a ribozyme induces rapid apoptosis of cancer cells through a telomere length-independent pathway. Cancer Biol Ther 4: 1098-1103, 2005.

28. Sachsinger J, Gonzalez-Suarez E, Samper E, Heicappell R, Müller M and Blasco MA: Telomerase inhibition in RenCa, a murine tumor cell line with short telomeres, by overexpression of a dominant negative mTERT mutant, reveals fundamental differences in telomerase regulation between human and murine cells. Cancer Res 61: 5580-5586, 2001.

29. Shay JW, Zou Y, Hiyama E and Wright WE: Telomerase and cancer. Hum Mol Genet 10: 677-685, 2001.

30. Massard C, Zermati Y, Pauleau AL, Larochette N, et al: hTERT: a novel endogenous inhibitor of the mitochondrial cell death pathway. Oncogene 25: 4505-4514, 2006.

31. Kurvinen K, Syrjanen S and Johansson B: Long-term suppression of telomerase expression in HeLa cell clones, transfected with an expression vector carrying siRNA targeting hTERT mRNA. Int J Oncol 29: 279-288, 2006.

32. Gambhir SS, Herschman HR, Cherry SR, et al: Imaging transgene expression with radionuclide imaging technologies. Neoplasia 2: 118-138, 2000.

33. Northcott PA, Jones DT, Kool M, et al: Medulloblastomics: the end of the beginning. Nat Rev Cancer 12: 818-834, 2012.

34. Stefani AL, Barzon L, Castagliuolo I, et al: Systemic efficacy of combined suicide/cytokine gene therapy in a murine model of hepatocellular carcinoma. J Hepatol 42: 728-735, 2005.

35. Ahn YH, Yi H, Shin JY, et al: STAT3 silencing enhances the efficacy of the HSV.tk suicide gene in gastrointestinal cancer therapy. Clin Exp Metastasis 29: 359-369, 2012.

36. Trinh QT, Austin EA, Murray DM, Knick VC and Huber BE: Enzyme/prodrug gene therapy: comparison of cytosine deaminase/5-fluorocytosine versus thymidine kinase/ganciclovir enzyme/prodrug systems in a human colorectal carcinoma cell line. Cancer Res 55: 4808-4812, 1995.

37. Bridgewater JA, Springer CJ, Knox RJ, Minton NP, Michael NP and Collins MK: Expression of the bacterial nitroreductase enzyme in mammalian cells renders them selectively sensitive to killing by the prodrug CB1954. Eur J Cancer 31: A2362-A2370, 1995.

38. Danks MK, Morton CL, Pawlik CA and Potter PM: Overexpression of a rabbit liver carboxylesterase sensitizes human tumor cells to CPT-11. Cancer Res 58: 20-22, 1998.

39. Boerman OC, Oyen WJ and Corstens FH: Progress in gene therapy: seeing is believing. J Nucl Med 42: 1235-1237, 2001.

40. Huang TG, Savontaus MJ, Shinozaki K, Sauter BV and Woo SL: Telomerase-dependent oncolytic adenovirus for cancer treatment. Gene Ther 10: 1241-1247, 2003.

41. Nishihara E, Nagayama Y,Mawatari F, et al: Retrovirus-mediated herpes simplex virus thymidine kinase gene transduction renders human thyroid carcinoma cell lines sensitive to ganciclovir and radiation in vitro and in vivo. Endocrinology 138: 4577-4583, 1997. 